Molecular Orbital Studies of Zinc Oxide Chemical Vapor Deposition Supplemental Material

Barbara H. Munk, Stanley M. Smith and H. Bernhard Schlegel*

Department of Chemistry, Wayne State University, Detroit, Michigan 48202 
Geometries Optimized at B3LYP/6-311G(d)

Structure 1: $\mathrm{Zn}\left(\mathrm{C}_{2} \mathrm{H}_{5}\right)_{2}$

-1937.7892696000 Hartrees

Input orientation:

\begin{tabular}{|c|c|c|c|c|}
\hline \multirow{2}{*}{$\begin{array}{l}\text { Center } \\
\text { Number }\end{array}$} & \multirow{2}{*}{$\begin{array}{l}\text { Atomic } \\
\text { Number }\end{array}$} & \multicolumn{3}{|c|}{ Coordinates (Angstroms) } \\
\hline & & $\mathrm{X}$ & $\mathrm{Y}$ & $\mathrm{Z}$ \\
\hline \multicolumn{5}{|c|}{ - - - - - - - - - - - - - - - - - - - - - - - - - - - - - - - - - - - - - - - - - - - - - - - - - } \\
\hline 1 & 30 & 0.000000 & -0.069750 & 0.000000 \\
\hline 2 & 6 & 0.028157 & -0.064782 & 1.953110 \\
\hline 3 & 6 & 1.417814 & 0.173337 & 2.567541 \\
\hline 4 & 1 & -0.375576 & -1.017487 & 2.316281 \\
\hline 5 & 1 & -0.668979 & 0.700672 & 2.314550 \\
\hline 6 & 1 & 1.392001 & 0.170388 & 3.663665 \\
\hline 7 & 1 & 2.137637 & -0.595036 & 2.266708 \\
\hline 8 & 1 & 1.841784 & 1.136377 & 2.264478 \\
\hline 9 & 6 & -0.028157 & -0.064782 & -1.953110 \\
\hline 10 & 6 & -1.417814 & 0.173337 & -2.567541 \\
\hline 11 & 1 & 0.375576 & -1.017487 & -2.316281 \\
\hline 12 & 1 & 0.668979 & 0.700672 & -2.314550 \\
\hline 13 & 1 & -1.392001 & 0.170388 & -3.663665 \\
\hline 14 & 1 & -2.137637 & -0.595036 & -2.266708 \\
\hline 15 & 1 & -1.841784 & 1.136377 & -2.264478 \\
\hline
\end{tabular}

Structure 2: HO-H-C ${ }_{2} \mathrm{H}_{5}$ TS

-155.593875495 Hartrees

Input orientation:

\begin{tabular}{|c|c|c|c|c|}
\hline \multirow{2}{*}{$\begin{array}{l}\text { Center } \\
\text { Number }\end{array}$} & \multirow{2}{*}{$\begin{array}{l}\text { Atomic } \\
\text { Number }\end{array}$} & \multicolumn{3}{|c|}{ Coordinates (Angstroms) } \\
\hline & & $\mathrm{X}$ & $\mathrm{Y}$ & Z \\
\hline \multicolumn{5}{|c|}{ - - - - - - - - - - - - - - - - - - - - - - - - - - - - - - - - - - - - - - - - - - - - - - - - - - - - - } \\
\hline 1 & 8 & 0.230477 & 0.397990 & -0.091931 \\
\hline 2 & 1 & 0.091649 & -0.316289 & 0.983292 \\
\hline 3 & 1 & 0.936692 & 1.005341 & 0.185452 \\
\hline 4 & 1 & -0.863005 & -0.919432 & 2.407696 \\
\hline 5 & 6 & 0.161871 & -0.961227 & 2.034983 \\
\hline 6 & 6 & 0.658694 & -2.343086 & 1.680418 \\
\hline 7 & 1 & 0.831056 & -0.391098 & 2.681591 \\
\hline 8 & 1 & -0.018879 & -2.847052 & 0.987544 \\
\hline 9 & 1 & 1.646131 & -2.305546 & 1.214170 \\
\hline 10 & 1 & 0.741206 & -2.970691 & 2.576704 \\
\hline
\end{tabular}


Geometries Optimized at B3LYP/6-311G(d)

\section{Structure 4: $\mathrm{Zn}(\mathrm{OH}) \mathrm{C}_{2} \mathrm{H}_{5} * * * \mathrm{C}_{2} \mathrm{H}_{5} \quad \mathbf{- 2 0 1 3 . 5 8 6 9 3 2 8 7 0 0}$ Hartrees}

Input orientation:

\begin{tabular}{|c|c|c|c|c|}
\hline \multirow{2}{*}{$\begin{array}{l}\text { Center } \\
\text { Number }\end{array}$} & \multirow{2}{*}{$\begin{array}{l}\text { Atomic } \\
\text { Number }\end{array}$} & \multicolumn{3}{|c|}{ Coordinates (Angstroms) } \\
\hline & & $\mathrm{x}$ & $\mathrm{Y}$ & Z \\
\hline 1 & 30 & 1.290080 & -0.686238 & 0.030521 \\
\hline 2 & 6 & 0.171764 & 0.140195 & 1.386360 \\
\hline 3 & 6 & 0.756810 & 0.100400 & 2.805247 \\
\hline 4 & 1 & -0.804924 & -0.354888 & 1.367388 \\
\hline 5 & 1 & -0.016499 & 1.177150 & 1.086601 \\
\hline 6 & 1 & 0.092814 & 0.581943 & 3.533397 \\
\hline 7 & 1 & 0.921565 & -0.923859 & 3.153123 \\
\hline 8 & 1 & 1.721862 & 0.612845 & 2.867767 \\
\hline 9 & 6 & 0.072803 & -0.360742 & -2.050209 \\
\hline 10 & 6 & -1.385324 & -0.126366 & -1.836913 \\
\hline 11 & 1 & 0.682587 & 0.476743 & -2.382771 \\
\hline 12 & 1 & 0.410853 & -1.328048 & -2.404133 \\
\hline 13 & 1 & -1.907119 & -0.023277 & -2.801839 \\
\hline 14 & 1 & -1.866490 & -0.956695 & -1.313173 \\
\hline 15 & 1 & -1.583857 & 0.789157 & -1.273994 \\
\hline 16 & 8 & 2.572683 & -1.611195 & -0.835907 \\
\hline 17 & 1 & 3.157547 & -1.141096 & -1.434600 \\
\hline
\end{tabular}

Structure 5: $\mathrm{Zn}(\mathrm{OH}) \mathrm{C}_{2} \mathrm{H}_{5}$

-1934.3978081800 Hartrees

Input orientation:

\begin{tabular}{|c|c|c|c|c|}
\hline \multirow{2}{*}{$\begin{array}{l}\text { Center } \\
\text { Number }\end{array}$} & \multirow{2}{*}{$\begin{array}{l}\text { Atomic } \\
\text { Number }\end{array}$} & \multicolumn{3}{|c|}{ Coordinates (Angstroms) } \\
\hline & & $\mathrm{X}$ & $\mathrm{Y}$ & $\mathrm{Z}$ \\
\hline \multicolumn{5}{|c|}{ - - - - - - - - - - - - - - - - - - - - - - - - - - - - - - - - - - - - - - - - - - - - - - - - - - - - - - - - - - - - - - } \\
\hline 1 & 30 & -0.223679 & -0.002115 & -0.413706 \\
\hline 2 & 6 & -0.230982 & -0.003883 & 1.510010 \\
\hline 3 & 6 & 1.165745 & -0.001016 & 2.151383 \\
\hline 4 & 1 & -0.795809 & -0.876845 & 1.854236 \\
\hline 5 & 1 & -0.797380 & 0.867710 & 1.856201 \\
\hline 6 & 1 & 1.107844 & 0.005634 & 3.245583 \\
\hline 7 & 1 & 1.750221 & -0.881614 & 1.868681 \\
\hline 8 & 1 & 1.751923 & 0.875151 & 1.858207 \\
\hline 9 & 8 & -0.147944 & -0.051740 & -2.192691 \\
\hline 10 & 1 & -0.731466 & 0.516716 & -2.698555 \\
\hline
\end{tabular}


Geometries Optimized at B3LYP/6-311G(d)

Structure 8: $\mathrm{Zn}(\mathrm{OH})_{2} \mathrm{C}_{2} \mathrm{H}_{5}$ -2010.1878937500 Hartrees

Input orientation:

\begin{tabular}{|c|c|c|c|c|}
\hline \multirow{2}{*}{$\begin{array}{l}\text { Center } \\
\text { Number }\end{array}$} & \multirow{2}{*}{$\begin{array}{l}\text { Atomic } \\
\text { Number }\end{array}$} & \multicolumn{3}{|c|}{ Coordinates (Angstroms) } \\
\hline & & $\mathrm{X}$ & Y & $\mathrm{Z}$ \\
\hline \multicolumn{5}{|c|}{ - - - - - - - - - - - - - - - - - - - - - - - - - - - - - - - - - - - - - - - - - - - - - - - - - } \\
\hline 1 & 30 & -1.295954 & -0.121165 & -0.001339 \\
\hline 2 & 6 & -0.217199 & -1.734782 & 0.065367 \\
\hline 3 & 6 & 1.296719 & -1.492353 & -0.014540 \\
\hline 4 & 1 & -0.537576 & -2.390123 & -0.751501 \\
\hline 5 & 1 & -0.463555 & -2.271006 & 0.988593 \\
\hline 6 & 1 & 1.862598 & -2.430789 & 0.031247 \\
\hline 7 & 1 & 1.584177 & -0.992146 & -0.944286 \\
\hline 8 & 1 & 1.657364 & -0.863513 & 0.805416 \\
\hline 9 & 8 & -1.869919 & 1.578109 & -0.186661 \\
\hline 10 & 1 & -2.248606 & 2.024031 & 0.574419 \\
\hline 11 & 8 & -3.599845 & -1.091771 & -0.004844 \\
\hline 12 & 1 & -3.920299 & -0.186835 & -0.004844 \\
\hline
\end{tabular}

Structure 9: $\mathrm{Zn}(\mathrm{OH})_{2} \mathrm{C}_{2} \mathrm{H}_{5} \mathrm{TS}$

-2010.1764119500 Hartrees

Input orientation:

\begin{tabular}{|c|c|c|c|c|}
\hline \multirow{2}{*}{$\begin{array}{l}\text { Center } \\
\text { Number }\end{array}$} & \multirow{2}{*}{$\begin{array}{l}\text { Atomic } \\
\text { Number }\end{array}$} & \multicolumn{3}{|c|}{ Coordinates (Angstroms) } \\
\hline & & $\mathrm{X}$ & $\mathrm{Y}$ & $\mathrm{Z}$ \\
\hline----- & ------ & --------1 & --------- & ------------- \\
\hline 1 & 30 & 0.140044 & 0.016525 & 0.091317 \\
\hline 2 & 6 & 0.016223 & -0.042436 & 2.079541 \\
\hline 3 & 6 & 1.385894 & 0.076349 & 2.702900 \\
\hline 4 & 1 & -0.454946 & -1.010041 & 2.274867 \\
\hline 5 & 1 & -0.672498 & 0.765684 & 2.343696 \\
\hline 6 & 1 & 1.345146 & -0.128999 & 3.782177 \\
\hline 7 & 1 & 2.087012 & -0.634504 & 2.258574 \\
\hline 8 & 1 & 1.809785 & 1.075967 & 2.577942 \\
\hline 9 & 8 & 1.851891 & -0.769076 & -0.221921 \\
\hline 10 & 1 & 2.071773 & -0.676169 & -1.159107 \\
\hline 11 & 8 & -0.594097 & 0.489953 & -1.484771 \\
\hline 12 & 1 & -1.075526 & -0.128936 & -2.038064 \\
\hline
\end{tabular}


Geometries Optimized at B3LYP/6-311G(d)

\section{Structure 10: $\mathrm{Zn}(\mathrm{OH})_{2}{ }^{* *} \mathrm{C}_{2} \mathrm{H}_{5} \quad \mathbf{- 2 0 1 0 . 1 9 7 5 7 3 3 1 0 0 ~ H a r t r e e s}$}

Input orientation:

\begin{tabular}{rrrrr} 
Center & Atomic & \multicolumn{3}{c}{ Coordinates } \\
Number & Number & $\mathrm{X}$ & $\mathrm{Y}$ & $\mathrm{Z}$ \\
-1 & 8 & -0.394920 & 1.872269 & -0.157207 \\
2 & 1 & -0.162271 & 2.373713 & 0.627435 \\
3 & 8 & -1.270727 & -1.552639 & -0.056048 \\
4 & 1 & -1.027561 & -2.106746 & -0.801645 \\
5 & 30 & -0.604889 & 0.103581 & 0.007104 \\
6 & 1 & 1.876047 & -0.991405 & -1.265696 \\
7 & 6 & 2.224642 & -0.261569 & -0.528875 \\
8 & 6 & 1.508651 & -0.403327 & 0.777771 \\
9 & 1 & 2.089443 & 0.739013 & -0.945662 \\
10 & 1 & 3.306322 & -0.426001 & -0.410729 \\
11 & 1 & 1.306859 & -1.397227 & 1.161273 \\
12 & 1 & 1.675128 & 0.367417 & 1.524739
\end{tabular}

Structure 11: $\mathrm{Zn}(\mathrm{OH})_{2} \quad-\mathbf{- 1 9 3 0 . 9 9 8 3 0 6 9 9 0 0 ~ H a r t r e e s}$

Input orientation:

\begin{tabular}{ccccc} 
Center & Atomic & \multicolumn{3}{c}{ Coordinates (Angstroms) } \\
Number & Number & $\mathrm{X}$ & $\mathrm{Y}$ & $\mathrm{Z}$ \\
-1 & 30 & -0.000016 & -0.002954 & -0.002196 \\
2 & 8 & -1.756171 & -0.106079 & 0.132209 \\
3 & 8 & 1.756047 & 0.083519 & -0.148975 \\
4 & 1 & -2.280099 & 0.508324 & -0.385972 \\
5 & 1 & 2.281579 & -0.239233 & 0.585973 \\
- & & & &
\end{tabular}


Geometries Optimized at B3LYP/6-311G(d)

\section{Structure 12: $\mathrm{Zn}\left(\mathrm{C}_{2} \mathrm{H}_{5}\right)_{2}{ }^{* *} \mathrm{H} 2 \mathrm{O} \quad$-2014.236222 Hartrees}

Input orientation:

\begin{tabular}{|c|c|c|c|c|}
\hline \multirow{2}{*}{$\begin{array}{l}\text { Center } \\
\text { Number }\end{array}$} & \multirow{2}{*}{$\begin{array}{l}\text { Atomic } \\
\text { Number }\end{array}$} & \multicolumn{3}{|c|}{ Coordinates (Angstroms) } \\
\hline & & $\mathrm{X}$ & $\mathrm{Y}$ & Z \\
\hline 1 & 30 & 0.195202 & -0.026483 & 0.032503 \\
\hline 2 & 6 & 0.458172 & -0.137735 & 1.977508 \\
\hline 3 & 6 & 1.924809 & -0.191086 & 2.435872 \\
\hline 4 & 1 & -0.072034 & -1.021020 & 2.358677 \\
\hline 5 & 1 & -0.037730 & 0.719910 & 2.452267 \\
\hline 6 & 1 & 2.027915 & -0.253906 & 3.526804 \\
\hline 7 & 1 & 2.451342 & -1.056539 & 2.018958 \\
\hline 8 & 1 & 2.484492 & 0.694805 & 2.116621 \\
\hline 9 & 6 & 0.406604 & 0.050684 & -1.922543 \\
\hline 10 & 6 & -0.885404 & 0.190370 & -2.740790 \\
\hline 11 & 1 & 0.943695 & -0.847233 & -2.254471 \\
\hline 12 & 1 & 1.078397 & 0.882188 & -2.171785 \\
\hline 13 & 1 & -0.701367 & 0.227963 & -3.821674 \\
\hline 14 & 1 & -1.567301 & -0.651735 & -2.571866 \\
\hline 15 & 1 & -1.430793 & 1.107407 & -2.487341 \\
\hline 16 & 8 & -2.134354 & 0.143687 & 0.129787 \\
\hline 17 & 1 & -2.731181 & 0.224895 & -0.619795 \\
\hline 18 & 1 & -2.651759 & 0.144854 & 0.939933 \\
\hline
\end{tabular}


Geometries Optimized at B3LYP/6-311G(d)

Structure 13: $\mathrm{Zn}\left(\mathrm{C}_{2} \mathrm{H}_{5}\right)_{2}{ }^{* *} \mathrm{HO}-\mathrm{H}-\mathrm{C}_{2} \mathrm{H}_{5} \mathrm{TS}$ -2093.40024566 Hartrees

\begin{tabular}{|c|c|c|c|c|}
\hline \multirow{2}{*}{$\begin{array}{l}\text { Center } \\
\text { Number }\end{array}$} & \multirow{2}{*}{$\begin{array}{l}\text { Atomic } \\
\text { Number }\end{array}$} & \multicolumn{3}{|c|}{ Coordinates (Angstroms) } \\
\hline & & $\mathrm{X}$ & $\mathrm{Y}$ & $\mathrm{Z}$ \\
\hline------ & ---- & --------- & ---------- & --------- \\
\hline 1 & 30 & -0.316862 & -0.173593 & -0.236098 \\
\hline 2 & 6 & -0.347671 & 0.341182 & 1.704049 \\
\hline 3 & 6 & 1.080649 & 0.348367 & 2.258964 \\
\hline 4 & 1 & -0.973025 & -0.363787 & 2.262023 \\
\hline 5 & 1 & -0.806301 & 1.330474 & 1.807545 \\
\hline 6 & 1 & 1.110426 & 0.630020 & 3.320655 \\
\hline 7 & 1 & 1.558090 & -0.633635 & 2.179839 \\
\hline 8 & 1 & 1.725704 & 1.054993 & 1.726894 \\
\hline 9 & 6 & 0.503051 & -0.658203 & -1.955046 \\
\hline 10 & 6 & -0.295647 & -0.197927 & -3.184481 \\
\hline 11 & 1 & 0.624026 & -1.748457 & -1.994237 \\
\hline 12 & 1 & 1.522486 & -0.257643 & -2.010285 \\
\hline 13 & 1 & 0.177896 & -0.493966 & -4.129408 \\
\hline 14 & 1 & -1.306394 & -0.619339 & -3.191578 \\
\hline 15 & 1 & -0.411215 & 0.891016 & -3.215494 \\
\hline 16 & 8 & -2.414781 & -0.105571 & -0.343625 \\
\hline 17 & 1 & -3.339916 & -0.300921 & -1.129970 \\
\hline 18 & 1 & -2.853383 & 0.130855 & 0.484384 \\
\hline 19 & 6 & -4.400769 & -0.437860 & -1.898055 \\
\hline 20 & 6 & -4.555385 & 0.839155 & -2.681868 \\
\hline 21 & 1 & -5.205745 & -0.657311 & -1.195006 \\
\hline 22 & 1 & -4.127078 & -1.319319 & -2.479604 \\
\hline 23 & 1 & -3.677569 & 1.041882 & -3.299661 \\
\hline 24 & 1 & -5.421457 & 0.782002 & -3.355813 \\
\hline 25 & 1 & -4.714308 & 1.700324 & -2.027425 \\
\hline
\end{tabular}


Geometries Optimized at B3LYP/6-311G(d)

Structure 14: $\mathrm{Zn}\left(\mathrm{C}_{2} \mathrm{H}_{5}\right) \mathrm{O}$

-3788.962195 Hartrees

Input orientation:

\begin{tabular}{|c|c|c|c|c|}
\hline \multirow{2}{*}{$\begin{array}{l}\text { Center } \\
\text { Number }\end{array}$} & \multirow{2}{*}{$\begin{array}{l}\text { Atomic } \\
\text { Number }\end{array}$} & \multicolumn{3}{|c|}{ Coordinates (Angstroms) } \\
\hline & & $\mathrm{x}$ & $\mathrm{Y}$ & Z \\
\hline 1 & 30 & 0.190265 & 0.082721 & -0.282193 \\
\hline 2 & 30 & 0.456486 & -0.021278 & 3.026050 \\
\hline 3 & 8 & 0.622760 & 0.124229 & -1.997186 \\
\hline 4 & 1 & -0.093864 & 0.109581 & -2.634810 \\
\hline 5 & 8 & -0.206246 & 0.040260 & 1.404923 \\
\hline 6 & 6 & 1.128362 & -0.105261 & 4.825861 \\
\hline 7 & 6 & 1.666296 & -1.483849 & 5.240520 \\
\hline 8 & 1 & 0.327062 & 0.198260 & 5.508599 \\
\hline 9 & 1 & 1.914743 & 0.648409 & 4.944623 \\
\hline 10 & 1 & 2.033709 & -1.483795 & 6.273011 \\
\hline 11 & 1 & 0.898405 & -2.260961 & 5.176584 \\
\hline 12 & 1 & 2.498182 & -1.809138 & 4.607861 \\
\hline
\end{tabular}

Structure 15: HOZnO

-1930.315548 Hartrees

Input orientation:

\begin{tabular}{ccccc} 
Center & Atomic & \multicolumn{2}{c}{ Coordinates (Angstroms) } \\
Number & Number & $\mathrm{X}$ & $\mathrm{Y}$ & $\mathrm{Z}$ \\
$-\ldots$ & 0.030740 & 0.000000 & 0.009955 \\
1 & 30 & 0.024465 & 0.000000 & 1.771560 \\
2 & 8 & 0.868618 & 0.000000 & 2.227509 \\
3 & 1 & -0.018887 & 0.000000 & -1.778570 \\
4 & 8 & &
\end{tabular}


Geometries Optimized at B3LYP/6-311G(d)

\section{Structure 16: $\left(\mathrm{C}_{2} \mathrm{H}_{5}\right) \mathrm{ZnOZnC} \mathrm{C}_{2} \mathrm{H}_{5} \quad \mathbf{- 3 7 9 2 . 3 5 9 9 4 3 ~ H a r t r e e s}$}

Input orientation:

\begin{tabular}{|c|c|c|c|c|}
\hline \multirow{2}{*}{$\begin{array}{l}\text { Center } \\
\text { Number }\end{array}$} & \multirow{2}{*}{$\begin{array}{l}\text { Atomic } \\
\text { Number }\end{array}$} & \multicolumn{3}{|c|}{ Coordinates (Angstroms) } \\
\hline & & $\mathrm{X}$ & $\mathrm{Y}$ & Z \\
\hline \multicolumn{5}{|c|}{ 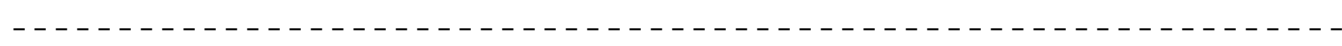 } \\
\hline 1 & 30 & 1.649586 & 0.079237 & -0.062891 \\
\hline 2 & 30 & -1.690393 & -0.318414 & -0.019287 \\
\hline 3 & 8 & 0.019688 & -0.417459 & 0.312389 \\
\hline 4 & 6 & -3.584209 & -0.221354 & -0.340811 \\
\hline 5 & 6 & -4.283777 & 0.965882 & 0.339972 \\
\hline 6 & 1 & -4.043988 & -1.158500 & -0.007183 \\
\hline 7 & 1 & -3.759868 & -0.179915 & -1.421901 \\
\hline 8 & 1 & -5.360075 & 0.981084 & 0.132733 \\
\hline 9 & 1 & -4.170177 & 0.940076 & 1.428246 \\
\hline 10 & 1 & -3.882503 & 1.927638 & 0.004534 \\
\hline 11 & 6 & 3.467420 & 0.588732 & -0.431068 \\
\hline 12 & 6 & 4.512751 & -0.144295 & 0.424813 \\
\hline 13 & 1 & 3.568970 & 1.670453 & -0.286797 \\
\hline 14 & 1 & 3.676438 & 0.415272 & -1.492923 \\
\hline 15 & 1 & 5.534197 & 0.172393 & 0.183831 \\
\hline 16 & 1 & 4.367591 & 0.036601 & 1.494389 \\
\hline 17 & 1 & 4.473677 & -1.228611 & 0.281490 \\
\hline
\end{tabular}

Structure 17: $\mathrm{HOZnOZnC}_{2} \mathrm{H}_{5}$

-1933.715395 Hartrees

Input orientation:

\begin{tabular}{|c|c|c|c|c|}
\hline \multirow{2}{*}{$\begin{array}{l}\text { Center } \\
\text { Number }\end{array}$} & \multirow{2}{*}{$\begin{array}{l}\text { Atomic } \\
\text { Number }\end{array}$} & \multicolumn{3}{|c|}{ Coordinates (Angstroms) } \\
\hline & & $\mathrm{X}$ & $\mathrm{Y}$ & $\mathrm{Z}$ \\
\hline------ & ------ & -------- & -------- & -------- \\
\hline 1 & 30 & 0.003467 & -0.749133 & -0.030395 \\
\hline 2 & 8 & 1.819140 & -0.743492 & -0.047888 \\
\hline 3 & 6 & -1.921948 & -0.760636 & 0.005265 \\
\hline 4 & 6 & -2.529447 & -1.194336 & 1.348971 \\
\hline 5 & 1 & -2.277699 & -1.420294 & -0.793069 \\
\hline 6 & 1 & -2.277692 & 0.241276 & -0.256772 \\
\hline 7 & 1 & -3.624443 & -1.183878 & 1.316581 \\
\hline 8 & 1 & -2.229672 & -2.208578 & 1.629509 \\
\hline 9 & 1 & -2.229667 & -0.535492 & 2.169525 \\
\hline
\end{tabular}


Geometries Optimized at B3LYP/6-311G(d)

\section{Structure 18: HOZnOZnOH}

\section{-3785.563332 Hartrees}

Input orientation:

\begin{tabular}{|c|c|c|c|c|}
\hline \multirow{2}{*}{$\begin{array}{l}\text { Center } \\
\text { Number }\end{array}$} & \multirow{2}{*}{$\begin{array}{l}\text { Atomic } \\
\text { Number }\end{array}$} & \multicolumn{3}{|c|}{ Coordinates (Angstroms) } \\
\hline & & $\mathrm{X}$ & Y & Z \\
\hline \multicolumn{5}{|c|}{ 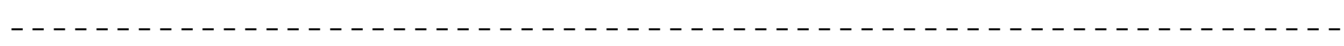 } \\
\hline 1 & 30 & 0.241957 & 0.014565 & -0.075448 \\
\hline 2 & 30 & 0.239673 & -0.012579 & 3.208831 \\
\hline 3 & 8 & 0.839976 & 0.012686 & -1.738133 \\
\hline 4 & 1 & 0.189785 & 0.038319 & -2.443186 \\
\hline 5 & 8 & -0.330905 & 0.017733 & 1.566411 \\
\hline 6 & 8 & 0.835352 & -0.045152 & 4.872038 \\
\hline 7 & 1 & 0.184266 & -0.028896 & 5.576546 \\
\hline
\end{tabular}

Structure 19: cyclic $\left(\mathrm{Zn}(\mathrm{OH}) \mathrm{C}_{2} \mathrm{H}_{5}\right)_{2} \quad \mathbf{- 3 8 6 8 . 8 7 4 4 1 2 6 4}$ Hartrees

Input orientation:

\begin{tabular}{|c|c|c|c|c|}
\hline \multirow{2}{*}{$\begin{array}{l}\text { Center } \\
\text { Number }\end{array}$} & Atomic & \multicolumn{3}{|c|}{ Coordinates (Angstroms) } \\
\hline & Number & $\mathrm{X}$ & $\mathrm{Y}$ & $\mathrm{Z}$ \\
\hline \multicolumn{5}{|c|}{ - - - - - - - - - - - - - - - - - - - - - - - - - - - - - - - - - - - - - - - - - - - - - - - - - - } \\
\hline 1 & 30 & 0.000000 & 0.000000 & 0.000000 \\
\hline 2 & 8 & 0.000000 & 0.000000 & 1.933367 \\
\hline 3 & 1 & 0.739381 & 0.000000 & 2.536578 \\
\hline 4 & 30 & -1.918983 & -0.000106 & 2.299277 \\
\hline 5 & 8 & -1.918983 & -0.000106 & 0.365910 \\
\hline 6 & 1 & -2.658364 & -0.000111 & -0.237301 \\
\hline 7 & 6 & 1.132154 & 0.000063 & -1.577859 \\
\hline 8 & 6 & 0.328733 & 0.000018 & -2.886892 \\
\hline 9 & 1 & 1.797755 & -0.870379 & -1.551637 \\
\hline 10 & 1 & 1.797639 & 0.870593 & -1.551647 \\
\hline 11 & 1 & 0.972829 & 0.000054 & -3.774747 \\
\hline 12 & 1 & -0.321782 & -0.877416 & -2.965432 \\
\hline 13 & 1 & -0.321885 & 0.877376 & -2.965428 \\
\hline 14 & 6 & -3.051136 & -0.000169 & 3.877135 \\
\hline 15 & 6 & -2.247715 & -0.000125 & 5.186169 \\
\hline 16 & 1 & -3.716629 & -0.870694 & 3.850919 \\
\hline 17 & 1 & -3.716730 & 0.870278 & 3.850918 \\
\hline 18 & 1 & -2.891812 & -0.000160 & 6.074024 \\
\hline 19 & 1 & -1.597106 & -0.877489 & 5.264710 \\
\hline 20 & 1 & -1.597192 & 0.877304 & 5.264704 \\
\hline
\end{tabular}


Geometries Optimized at B3LYP/6-311G(d)

\section{Structure 20: cyclic $\left(\mathrm{Zn}(\mathrm{OH})_{2}\right)_{2} \quad \mathbf{- 3 8 6 2 . 0 7 7 6 7 2 2 5}$ Hartrees}

Input orientation:

\begin{tabular}{|c|c|c|c|c|}
\hline \multirow{2}{*}{$\begin{array}{l}\text { Center } \\
\text { Number }\end{array}$} & \multirow{2}{*}{$\begin{array}{l}\text { Atomic } \\
\text { Number }\end{array}$} & \multicolumn{3}{|c|}{ Coordinates (Angstroms) } \\
\hline & & $\mathrm{X}$ & $\mathrm{Y}$ & $\mathrm{Z}$ \\
\hline \multicolumn{5}{|c|}{ - - - - - - - - - - - - - - - - - - - - - - - - - - - - - - - - - - - - - - - - - - - - - - - - - - - - } \\
\hline 1 & 30 & 0.000000 & 0.000000 & 0.000000 \\
\hline 2 & 8 & 0.000000 & 0.000000 & 1.905912 \\
\hline 3 & 8 & 1.069414 & 0.000000 & -1.436683 \\
\hline 4 & 1 & 0.647344 & 0.000775 & -2.297763 \\
\hline 5 & 1 & 0.742099 & -0.000734 & 2.505304 \\
\hline 6 & 30 & -1.922591 & -0.000566 & 2.236788 \\
\hline 7 & 8 & -1.922589 & -0.000293 & 0.330878 \\
\hline 8 & 1 & -2.664684 & -0.001331 & -0.268519 \\
\hline 9 & 8 & -2.992032 & -0.001210 & 3.673451 \\
\hline 10 & 1 & -2.569979 & -0.000185 & 4.534538 \\
\hline
\end{tabular}

Structure 21: $\mathrm{Zn}\left(\mathrm{C}_{2} \mathrm{H}_{5}\right)_{2} * * \mathrm{H}_{2} \mathrm{O}$ TS $\quad \mathbf{- 2 0 1 4 . 1 8 8 9 0 9 9 4}$ Hartrees

Input orientation:

\begin{tabular}{|c|c|c|c|c|}
\hline \multirow{2}{*}{$\begin{array}{l}\text { Center } \\
\text { Number }\end{array}$} & \multirow{2}{*}{$\begin{array}{l}\text { Atomic } \\
\text { Number }\end{array}$} & \multicolumn{3}{|c|}{ Coordinates (Angstroms) } \\
\hline & & $\mathrm{X}$ & $\mathrm{Y}$ & $\mathrm{Z}$ \\
\hline \multicolumn{5}{|c|}{-------------------------------------------------------} \\
\hline 1 & 30 & 0.000000 & 0.000000 & 0.000000 \\
\hline 2 & 6 & 0.000000 & 0.000000 & 2.256632 \\
\hline 3 & 6 & 1.476076 & 0.000000 & 2.688873 \\
\hline 4 & 1 & -0.377807 & -1.032240 & 2.196397 \\
\hline 5 & 1 & -0.614370 & 0.420861 & 3.064130 \\
\hline 6 & 1 & 1.634837 & -0.434747 & 3.682009 \\
\hline 7 & 1 & 2.114193 & -0.562541 & 1.997260 \\
\hline 8 & 1 & 1.879841 & 1.018193 & 2.720824 \\
\hline 9 & 6 & 0.478751 & -1.220331 & -1.441581 \\
\hline 10 & 6 & 0.634019 & -0.562116 & -2.820093 \\
\hline 11 & 1 & -0.285245 & -2.004354 & -1.486783 \\
\hline 12 & 1 & 1.405465 & -1.734142 & -1.163570 \\
\hline 13 & 1 & 0.893628 & -1.292235 & -3.595753 \\
\hline 14 & 1 & -0.286586 & -0.066717 & -3.143485 \\
\hline 15 & 1 & 1.420006 & 0.199800 & -2.825618 \\
\hline 16 & 8 & -0.628938 & 1.822618 & 0.495275 \\
\hline 17 & 1 & -0.256887 & 2.700886 & 0.371488 \\
\hline 18 & 1 & -0.386368 & 1.189821 & 1.484969 \\
\hline
\end{tabular}


Geometries Optimized at B3LYP/6-311G(d)

\section{Structure 22: $\mathrm{Zn}(\mathrm{OH}) \mathrm{C}_{2} \mathrm{H}_{5} * * \mathrm{H}_{2} \mathrm{O}$ TS $\quad \mathbf{- 2 0 1 0 . 7 9 3 5 6 4 2 7}$ Hartrees}

Input orientation:

\begin{tabular}{ccccc} 
Center & Atomic & \multicolumn{3}{c}{ Coordinates (Angstroms) } \\
Number & Number & Y & $\mathrm{Z}$ \\
-1 & 30 & 0.000000 & 0.000000 & 0.000000 \\
2 & 6 & 0.000000 & 0.000000 & 2.201733 \\
3 & 6 & 1.531234 & 0.000000 & 2.386855 \\
4 & 1 & -0.467652 & -0.470469 & 3.076325 \\
5 & 1 & -0.394029 & 1.025750 & 2.240383 \\
6 & 1 & 1.853047 & 0.439768 & 3.336772 \\
7 & 1 & 1.929612 & -1.020017 & 2.356137 \\
8 & 8 & 0.835157 & 1.087496 & -1.152737 \\
9 & 1 & 0.706838 & 2.030932 & -1.032080 \\
10 & 1 & -0.876799 & -2.576297 & 0.225033 \\
11 & 1 & -0.965967 & -1.637287 & 0.417280 \\
12 & 1 & -0.629841 & -1.129686 & 1.444362 \\
13 & 1 & & &
\end{tabular}

\section{Structure: $\mathrm{C}_{2} \mathrm{H}_{5} * * \mathrm{H}_{2}$ TS}

\section{-80.3375662871 Hartrees}

Input orientation:

\begin{tabular}{|c|c|c|c|c|}
\hline \multirow{2}{*}{$\begin{array}{l}\text { Center } \\
\text { Number }\end{array}$} & \multirow{2}{*}{$\begin{array}{l}\text { Atomic } \\
\text { Number }\end{array}$} & \multicolumn{3}{|c|}{ Coordinates (Angstroms) } \\
\hline & & $\mathrm{X}$ & $\mathrm{Y}$ & $\mathrm{Z}$ \\
\hline \multicolumn{5}{|c|}{ 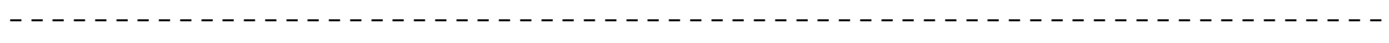 } \\
\hline 1 & 6 & 0.039146 & 0.023750 & 0.048469 \\
\hline 2 & 6 & -0.016030 & -0.145979 & 1.544091 \\
\hline 3 & 1 & 1.067078 & -0.008293 & -0.322955 \\
\hline 4 & 1 & -0.513946 & -0.774356 & -0.462996 \\
\hline 5 & 1 & -0.402043 & 0.973553 & -0.265657 \\
\hline 6 & 1 & -0.990189 & 0.001741 & 2.008648 \\
\hline 7 & 1 & 0.505303 & -1.012936 & 1.948206 \\
\hline 8 & 1 & 1.192634 & 1.580504 & 2.464871 \\
\hline 9 & 1 & 0.710258 & 0.891466 & 2.097389 \\
\hline
\end{tabular}

Structure: $\mathrm{H}^{* *} \mathrm{H}_{2} \mathrm{O}$ TS

-76.9194558788 Hartrees

Input orientation:

\begin{tabular}{ccccc} 
Center & Atomic & \multicolumn{3}{c}{ Coordinates } \\
Number & Number & $\mathrm{X}$ & $\mathrm{Y}$ & $\mathrm{Z}$ \\
$\mathrm{n}$ & 8 & 0.011747 & 0.000000 & 0.031835 \\
2 & 1 & -0.001161 & 0.000000 & 1.339029 \\
3 & 1 & 0.969537 & 0.000000 & -0.144338 \\
4 & 1 & 0.210669 & 0.000000 & 2.158919
\end{tabular}


Geometries Optimized at B3LYP/6-311G(d)

\section{Structure: HZnOH}

\section{-1855.73309633 Hartrees}

Input orientation:

\begin{tabular}{|c|c|c|c|c|}
\hline \multirow{2}{*}{$\begin{array}{l}\text { Center } \\
\text { Number }\end{array}$} & \multirow{2}{*}{$\begin{array}{l}\text { Atomic } \\
\text { Number }\end{array}$} & \multicolumn{3}{|c|}{ Coordinates (Angstroms) } \\
\hline & & $\mathrm{X}$ & $\mathrm{Y}$ & Z \\
\hline \multicolumn{5}{|c|}{ - - - - - - - - - - - - - - - - - - - - - - - - - - - - - - - - - - - - - - - - - - - - - - - } \\
\hline 1 & 30 & -0.004321 & 0.093851 & -0.030955 \\
\hline 2 & 1 & -1.499388 & 0.085062 & 0.028638 \\
\hline 3 & 8 & 1.768161 & 0.094133 & -0.032868 \\
\hline 4 & 1 & 2.236717 & 0.216119 & -0.860069 \\
\hline
\end{tabular}

Structure: $\mathrm{ZnOH}$

\section{-1855.09384860 Hartrees}

Input orientation:

$\begin{array}{ccccc}\text { Center } & \text { Atomic } & \text { Coordinates } & \text { C Angstroms ) } \\ \text { Number } & \text { Number } & \mathrm{X} & \mathrm{Y} & \mathrm{Z} \\ \mathrm{y} & 30 & -1.058246 & 0.270459 & -0.003874 \\ 2 & 8 & 0.858678 & 0.267641 & 0.000322 \\ 3 & 1 & 1.192702 & -0.239106 & 0.754757\end{array}$

